\title{
Sibylle Raasch Gleichstellung der Geschlechter oder Nachtarbeitsverbot für Frauen?
}

Das Nachtarbeitsverbot für Frauen wankt in Europa bercits seit längerem. Zusammen mit Belgien bildeten Deutschland (alte Bundesländer), Frankreich und Öster-

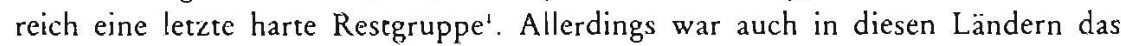
Verbot durchlöchert von quantitativ und qualitativ erheblichen Ausnahmen. Wo Nachtarbeit durch Frauen für die Wirtschaft wichtig ist, wie beispielsweise in der Gastronomie, im Unterhaltungsbereich oder in der Krankenpflegc, war sic faktisch auch in diesen Staaten zugclassen. 7,2\% aller beschäftigten Frauen leisteten 1989 in der alten Bundesrepublik Nachtarbeit, im Dienstleistungsbereich allein waren es schon ${ }_{4}, 8 \%$, bei jewcils steigender 'Tendenz ${ }^{2}$.

Jetzt liegen für Frankreich, mit Auswirkungen auch auf den gesamten Bereich der EG, sowie Deutschland und Österreich neue höchstrichterliche Entscheidungen vor, die sich bei vergleichbarer Fallkonstellation nach Begründung und Ergebnis erheblich unterscheiden: Im Juli I 991 entschied der Gcrichtshof der Europäischen Gemeinschaften ${ }^{3}$, Art. L $213^{-1}$ des französischen Code du travail sei als Hemmnis für den Wettbewerb freier Marktbürgerinnen und -bürger wegen Geschlechtsdiskriminierung nicht mehr anwendbar. Das deutsche Bundesverfassungsgericht ist dicsem Ergebnis für den Fall des $\$ 19$ Abs. x AZO im Januar 1992 gefolgt', jedoch nicht ohne bei dieser Gelcgenheit eine differcnziertere glcichstellungspolitische Position zu beziehen. In Kenntnis und unter Bezugnahme auf diese beiden Urteile hat danach im März 1992 der österreichische Verfassungsgerichtshof das dortige Frauennachtarbeitsgesetz dennoch als nicht frauendiskriminierend aufrechterhalten' und seine bereits in Juni 1988 bezogene Position eher patriarchalischer Protektion von Frauen $^{6}$ trotz der ansonsten wachsenden EG-Orientierung österreichischer Politik und Rechtsprechung ${ }^{7}$ erneut bekräfrigt.

Hauptfrage war in allen drei Entscheidungen, ob das Nachtarbeitsverbot frauendiskriminierend oder als Schutz der Frau gegen Doppelbelastung durch Nachtarbeit einerseits und Haus- und Erziehungsarbeit andererseits gerechtfertigt ist. Insofern erölfnet der Vergleich der drei Entscheidungen Perspektiven, wie Recht angesichts der vorherrschenden geschlechtsspezifischen Arbeitsteilung in der Gesellschaft mit dem Gleichberechtigungsanspruch der Frauen umgehen kann und soll, auch über die Nachtarbeitsproblematik hinaus. Im folgenden möchte ich daher untersuchen, wie

I Vgl. E. Waldherr: Das EuGH-Erkenntnis zum Nachtarbeitsverbot fur Frauen, in: Bundesmunisterium fur Arbeit und Soziales (Hg.): Niachearbcit, März, 1992, S. 73 fF., S. 74.

2 H. Seilert/B. Stolz-Willig: Nachtarbeit sozialvertraglich gestalten, in: WSI Mitteilungen 1992, S. is 8 ff. S. 159.

3 EuGH, Rs. C-345/89 vom 25.7.1991, EuGRZ 1991, S. $421 \mathrm{ff}$.

4 BVerfG, I BvR $1025 / 82$ u. a. vom 28. 1. 1992, EuGRZ 1992, S. 17ff.

s VfGH, G 220-222/91-9 vom 12. 3. 1992, in: Bundesministerium a. a, O., S. A $40 \mathrm{ff}$.

6 VfGH, B 806/807 vom 30.6.1988, VfSlg. $11.774 / 1988$, S. $865 \mathrm{ff}$. = EuGRZ 1992, S. $370 \mathrm{ff}$.

7 Vgl. VIGH, EuGRZ 1991, S. 491. 
dic drei Gerichte in ihren Entscheidungen die Problematik der geschlechrsspezifischen Arbeitsteilung wahrnehmen (1.). Vor dem Hintergrund der These, daß die hiermit zusammenhängende strukturelle Frauendiskriminierung mit den tradierten rechrlichen Diskriminierungsverboten nicht zu beseitigen ist (2.), sollen dann die gleichstellungsrechtlichen und frauenpolitischen Implikationen und Konsequenzen der drei Entscheidungen im Vergleich näher untcrsucht werden (3.).

\section{Die Nachtarbeitsfrage als Problem fortbestehender geschlechtsspezifischer Arbeitsteilung}

EuGH und BVerfG stimmen darin überein, daß Nachtarbcit für Frauen allgemein betrachtet ebenso schädlich ist wie für Männer, und auch der VfGI zieht die alte These von der besonderen biologischen Schutzbedürftigkeit der nachtarbeitenden Frau inzwischen zumindest in Zweifel. Dicse Rechtsprechung befinder sich in Einklang mit der ncuen arbcitsmedizinischen Literatur". Ein spczifisches gesundheitliches Schurzbedürfnis von Frauen vor Nachtarbeit wird in der Literatur vor allem damit begründet, daß bei Frauen durch Nachtarbeit nicht nur der Biorhythmus gestört werde, sondern sie zusätzlich im Gegensatz zu Männern noch der Doppelbelastung von Nachtarbeit und Haus- und Erziehungsarbeit unterlägen. Insbesondere das Schlafdefizit von Nachtarbeiterinnen, dic tagsüber Hausarbeit leisten und sich um ihre Kinder kümmern müßten, wird angeführt ${ }^{9}$.

Der EuGH stellt sich der Hausarbeitsproblematik in seinem Urteil nicht. Lakonisch formuliert er, daß die Richtlinie 76/207/EWG, an welcher er die Zulässigkeit des französischen Frauennachtarbeitsverbots mißt, nicht zum Gegenstand habe, »die internen Verhältnisse der Familie zu regeln oder die Aufgabenteilung zwischen den Eltern zu ändern ${ }^{10}$. Mit demselben Hinweis hatte der EuGH bereits 1984 dic Klage eines deutschen Vaters auf Mutterschaftsurlaub nach dem damaligen Mutterschutzgesetz zurückgewiesen'". Auch wenn die Stoßrichtung der EG-Richtlinie damit zutreffend wiedergegcben wird, ist dieses Vorgehen problematisch. Die scharfe Trennung, die der EuGH zwischen Privatbereich und Erwerbsbereich ziehen möchte, ist nicht aufrechtzuerhalten, wie unten (2.) genauer ausgeführt werden soll.

Der VfGH meint, "daß das traditionelle Rollenbild der Frau in Haushaltsführung und Kindererzichung nur allmählich einem partnerschaftlichen Verhalten weicht" und Frauen bisher die Hauptlast der Haushaltsführung und Kindererziehung... noch immer tragen ${ }^{12}{ }^{2}$. Dic Einschätzung des BVerfG erschcint demgegenüber differenzicrter und tendenzicll optimistischer. Die Doppelbelastung treffe die Frau in ihrer ganzen Schwere nur dann, wenn sie betrcuungsbedürftige Kinder habe und allein stche oder der Mann ihr trotz der Nachtarbeit Haushalt und Kinder überlasse. $\mathrm{Da}$ auch alleinerziehende Väter und partnerschaftlich lebende Paare der Doppelbelastung ausgesetzt seien, ginge es letztlich hier um ein "nicht zu leugnendes Schutzbedürnis für Nachtarbeiterinnen und Nachtarbeiter, die zugleich Kinder zu betreuen und cinen Mehrpersonenhaushalt zu führen« hätten. Dem aber könne sach-

$8 \mathrm{Vgl}$. G. Elsner: Nachtschichtarbeit ist grundsaczlich schadlich, in: AiB I992, S. I94 II.; B. Kupper/Br. Scolz-Willig: Frauenarbeitsschutz - noch zeitgema in einem veretnten Deurschland? in: WSI Mitteilungen 1991, S. 555 ff., S. 559 , jeweils m. w. N.

9 BVerfG, EuGRZ 1992, S. 21 m. w. N.; M. Dobberthien: Schutz der oder Schurz vor weiblicher Arbetskraft? in: WSI Mittelungen $198 \mathrm{r}$, S. 233 ff., S. 236 ; Kupper/Stolz-Willig, a. a. O., S. 560.

10 EuGH, EuGRZ 1991, S. 422.

II V.uGH, Slg. 1984 , S. 3047 ff., S. 3075 .

12 VIGH, Bundesmintsterium a.a.O., S. A $43 \mathrm{~m}$.w. N 
gerechter durch Regelungen Rechnung getragen werden, die an diesen Tatbestand anknüpften. Der soziale Befund reiche zur Reclatfertigung einer geschlechtsbezogenen Ungleichbehandlung nicht aus' ${ }^{13}$.

Damit allerdings eilt das BVerfG der realen Entwicklung zum Teil wcit voraus. Breit angelegte empirische Untersuchungen 1985 und $x 988$ kommen bezogen auf die Mitarbeit der Männer im Haus zu eher deprimierenden Ergebnissen: "92\% aller Männer, die mit ihrer Partnerin zusammenleben, fühlen sich durch Hausarbeit kaum belastet. Zu Recht: Sie tun so gut wic nichts"'4. "Der äußere Machtzuwachs der Frau, der mit eigenem Geld, Berufsstatus etc. verbunden ist, verändert in den heutigen Partnerschaften nicht unbedingt die inneren Entscheidungsstrukturen ... Die Männer... unterstützen die Berufstätigkeit ihrer Frau. Aber nur, solange es ihre Bequemlichkeit nicht schmerzlich tangiert und es dem Kind ihrem Urteil nach gutgeht «'s. Selbst bei Hausmännern mit vollzeitbeschäftigter Partnerin leistet die Frau noch immer fast die Hälfte der anfallenden Hausarbeit. ${ }^{16}$ Auch in der DDR konnte bei den Männern kein entscheidender Durchbruch zur Entlastung berufstätiger Frauen von Familienverantwortung erreicht werden ${ }^{17}$. Bei genauerer Betrachtung leisten Frauen Hausarbeit im übrigen keineswegs nur für ihre Kinder und pflegebedürftigen Angehörigen, sondern ebenso auch für ihre Männer, die "Ansprüche « häufig auch viel geschickter und nachdrücklicher einzufordern verstehen. Die Belastungsdiskrepanz zwischen Frauen und Männern ist damit noch größer: Der weiblichen Mehrarbeit für andere steht das männliche Entlastetsein durch andere heute noch als Regelfall gegenüber. Die alleinerziehenden Väter schließlich machten in den alten Bundesländern noch 1987 nur einen Anteil von $14 \%$ an allen Alleincrziehenden aus, in der DDR scheinen sie seit $198 \mathrm{I}$ mit nur $\mathrm{r} \%$ auch heute noch eine zu vernachlässigende Größc darzustellen's. Der durch Haus- und Erzichungsarbeit »wie eine Frau * belastete Mann hat also immer noch Seltenheitswert ebenso wie die durch ihren Mann partnerschaftlich entlastete Frau.

Dennoch entspricht die Situation der erwerbstätigen Frau mehrheitlich nicht mehr dem Bild der doppelt belasteten Hausfrau und Mutter, von dem das Nachtarbeitsverbot konzeptionell ausgeht. Mit sinkender Geburtenrate, stcigender Lebenserwartung und einer wachsenden Zahl auch jüngerer Alleinlebender kann heute nieht mehr pauschal unterstellt werden, jede Frau sei Hausfrau und Mutter. In der alten Bundesrepublik waren 1987 nur $55 \%$ der erwerbstätigen Frauen verheiratet und nur $22 \%$ hatten Kinder unter is Jahren. Seine reale Entsprechung findet das Frauenbild des Nachtarbeitsverbots vor allem bei der Gruppe der 35-bis 44-jährigen erwerbstätigen Frauen: Hier waren $198574 \%$ verheiratet, und $69,9 \%$ hatten mindestens cin lediges Kind in der Familie ${ }^{19}$. Der VfGH sieht diese Unterschiede zwischen Frauen in verschiedenen Lebenslagen, möchte cs denjenigen Frauen, ndie dieses Schutzes aufgrund ihrer günstigen Lage nicht (mehr) bedürfen «, jedoch zumuten, win Solidarität mit den Schutzbedürftigen auf Nachtarbeit zu verzichten ${ }^{20}$.

Für ein derartiges Solidaritätsopfer besteht jedoch keine Notwendigkeit, wenn, wie vom BVerfG vorgeschlagen, der Schutz an dic tatsächlich vorhandene Belastung mit durch Kinder bedingter Haus- und Erziehungsarbeit anknüpft. Der Lösungsweg des BVerfG ist zudem emanzipatorischer, wcil cr Frau-Sein und soziale Mutterschaft

13 BVerfG, EuGRZ 1992, S. 21 .

14 S. Metz-Göckel/U. Muller: Der Mann, 1985, S. 24 m.w. N

is G. Erler u. a.: Kind? Beruf? oder beides? 1988, S. 56 If.

I6 B. Strumpel u. a.: Teilzcitarbeitende Manner und Hausmanner, 1988, 5. 9 und $12 \mathrm{ff}$.

17 G. Winkler (Hg.): Frauenreport'90, 1990, S.81.

18 BMJFFG (Hg.) Frauen in der Bundesrepublik Deutschland, 1989, S. 59 ; Winkler a. a. O., S. 1 1 2 f.

19 BMJFFG a. a. O., S. 32 ; Stat. Bundesamt: Frauen in Famslie, Bcruf und Gesellschaft, 1987, S. 88.

20 VIGH, Bundesministerium 3. a. O., S. 143. 
nicht mehr gleichsetzt, sondern nach der tatsächlichen Rollenverteilung im Einzelfall fragt und offen dafür ist, das - derzeit leider noch ausstehende - familiäre Engagement von Männern einzubeziehen.

\section{Strukturelle Frauendiskriminierung und Gleichberechtigungsgebot}

Eine »strukturelle Frauendiskriminierung « begrifflich neben bzw. hinter die rechtsdogmatisch weitgehend umrissenen Fälle direkter und mittelbarer Diskriminierung ${ }^{21}$ zu stellen, geht auf das Benda-Gutachten zur Hamburger Frauenquote im öffentlichen Dienst 1986 zurück $^{22}$. Benda meint damit den Fall, daß durch zahlenmäßige Diskrepanzen zwischen den Geschlcchtern ungleiche Ergebnisse sichtbar werden, die auf eine Benachteiligung der Frauen hinweisen, ohne daß der Diskriminierungsvorgang für einzclne Frauen belcgt und konkrete Arbeitgeber als dafür verantwortliche Diskriminicrer sanktioniert werden können. Beispielsweise ist die Unterrepräsentanz von Frauen in sog. Männerberufen oder dem Führungsbereich nicht ausschließlich direkter und mittelbarcr Einstellungs- und Aufstiegsdiskriminierung geschuldet. Die Kausalkette reicht vielmehr grob vereinfacht von der frühkind'ichen Erziehung der Frauen zu Anpassungsbereitschaft und sozialer Mutterschaft über Schule und Hochschule, die Frauen immer noch auf dienende und helfende Berufe hin orientieren und keine Vorbilder für einflußreiche, durchsetzungsfähige Frauen liefern, über Partnerschaftskonzepte, nach denen die Frau immer noch kleiner, dem Mann zumindest ein wenig unterlegen und angepaßt zu sein hat, bis hin zu der Tatsache, daß in Männerberufen und Führungspositionen besonders wenig auf familiäre Orientierungen und Belastungen Rücksicht genommen wird. In dicsem Sinne ist Frauenbenachteiligung heute noch strukturell, gehört zum gesellschaftlichen Grundmuster und ist allein durch einzelfallbezogene Verbote direkter und mittclbarer Diskriminierung nicht zu beseitigen.

Das BAG hat diesen Unterschied zwischen mittelbarer und struktureller Diskriminierung bereits einmal im Fall der Privilegierung von männlichem Führungspersonal bei der betrieblichen Altersversorgung implizit deutlich gemacht. Im Gegensatz zum Fall der Benachteiligung von weiblichen Teilzeitkräften weigerte sich das BAG, eine mittelbare Diskriminierung anzunehmen, obwoh! Fraucn in den begünstigten Führungspositionen quantitativ ebenso deutlich unterrepräsentiert waren wie in der Teilzeitarbeit überrepräsentiert. Es sei "zu berücksichtigen, daß sich die negative Auswahl von weiblichen Arbeitnehmern in mehreren Schritten " vollziehe und nicht allein im Zusammenhang mit der umstrittenen Versorgungsrcgelung gewürdigt werden könne. Es sei keinc Besonderheit gerade eines einzelnen Betriebes, daß die Zahl der Frauen in Aufstiegspositionen weitaus geringer sei als ihr Anteil an der Gesamtbelegschaft ${ }^{23}$. Im Ergebnis beurteilte es die günstigere Altersversorgungsregelung der Männer in Führungspositionen als für sich betrachtet nicht diskriminierenden letzten Schritt in einer Abfolgekette, deren Ergebnis auf andere Weise zu korrigieren sei.

Innerhalb des Multifaktorenbündels struktureller Diskriminierung ist die einseitige Einbindung nur der Frauen in private Haus-, Erziehungs- und Pflegearbeit ein zentraler Kulminationspunkt. Hand in Hand als Kehrseite ein und derselben Medaille

\footnotetext{
21 Siehe hierzu ausfuhrlich H. M. Pfarr/Kl. Bertelsmann: Disknminierung im Enwerbsleben, 1989.

22 E. Benda: Notwendigkeit und Móglichkes positiver Akıonen zugunsten von Frauen im öffendichen Dienst, 1986, S. 7 und 33 . 23 BAG, DB 1987, S. 994 f.
} 
entstanden außerhäusliche Erwerbsarbeit des Mannes und Hausfrauendasein der Frau in der bürgerlichen Gesellschaft des $18 . / 19$. Jahrhunderts ${ }^{24}$. Das am männlichen Lebenszusammenhang orientierte heutige Normalarbeitsverhälınis, konzipiert als lebenslang, ganztags und unterbrechungslos, ist nur lebbar, wenn es unterstützt und flankiert wird durch ein nicht denselben $Z$ wängen des Erwerbslebens folgendes versorgendes Hausfrauendasein. Gerade die Einführung des Nachtarbeitsverbots trug mit dazu bei, das Konzept der "Hausfrau « und des "Familienlohns mitzuschaffen und zu verfestigen auch dort, wo die Frauen tatsächlich außer Haus arbeiteten ${ }^{\text {is. Wer }}$ Frauen als Gruppe ebenso wie Männern den Erwerbsbereich und die bezahlte Arbeit zugänglich machen will wie der EuGH, muß sich deshalb auch über die andere Hälfte der gesellschaftlich notwendigen Arbeit, die Frauen bisher allein leistcten, Gedanken machen.

Die tradierte ratio von Diskriminicrungsverboten war es bisher, den Mann zum Maßstab des Normalniveaus zu machen, von dem Fraucn als Diskriminierte wegen ihres Geschlechts ausgeschlossen sind. So sprach Dürig von der "Zielrichtung des Gleichberechtigungsgrundsatzes auf Angleichung der Frauenstellung an die Position des Mannes «" ${ }^{26}$. Die Diskriminierungsfeststellung kann nur im Vergleich zwischen den Geschlechtern geschehen, wobei die direkte Diskriminierung im unnittelbaren personalen Vergleich zwischen Mann und Frau und die mittelbare durch Relationen quantitativ unterschiedlicher Betroffenheit der Geschlechter erfolgt, insofern ist Dürig Recht zu geben. Die Folgerung, dic Beseitigung der Diskriminierung könnc deshalb auch nur so geschehen, die Frau auf das Niveau des Mannes zu heben, ist jedoch kurzschlüssig, wenn man sie auf die gesamte Gesellschaft und einen längeren Zeithorizont bezieht. Es muß auch um Umverteilung gehen. Im Rahmen der innergewcrkschaftlichen Auseinandersetzungen um Frauenlohndiskriminierung wurde das schon früh deutlich: Weil bei konstanter Lohnsumme letztlich nur eine Umverteilung der gesamten Lohnsumme zwischen beiden Geschlechtern Abhilfe verspricht, war die männliche Gewerkschaftsmehrheit bisher nic für einen nachdrücklichen Einsatz auf breiter Linie gegen Frauenlohndiskriminierung zu aktivieren ${ }^{27}$.

Gleichstellungspolitik, die Frauen die Erwerbsarbcit wie Männern zugänglich machen will, also tatsächliche Wettbewerbsfreiheit auf dem Arbeitsmarkt herstellen will, muß Strukturveränderungen sowohl am Normalarbeitsverhältnis als auch im familiären Bereich vornehmen. Schon deshalb greifen sowohl die EG-Gleichbehandlungsrichtlinien $76 / 207$ als auch der EuGH in seinem Bezug allein hierauf zu kurz, wenn sie sich auf den Erwerbsbereich beschränken. Gleichbehandlungspolitik kann langfristig nicht losgelöst von den faktischen und rechtlichen familiären Rahmenbedingungen betrieben werden ${ }^{28}$. Es zeigt die wachsende Sensibilität des BVerfG für den Zusammenhang von Haus- und Erwerbsarbeit, daß es sich zumindest in der Rentenfrage zunehmend derartigen Problemen öfnet ${ }^{29}$.

Allerdings handelt es sich hier um erste tastende Schritte. Ein nachhaltiger Schlag gegen strukturelle Frauendiskriminierung ließe sich nur führen, wenn das Arbeitsund Sozialrecht Haus- und Erwerbsarbeit miteinander kombiniert zum Normalfall erheben und clurch entsprechende flankierende Maßnahmen auch tatsächlich lebbar

24 Vgl. U. Frevert: Frauen-Geschichte, 1986; U. Gerhard: Verhaltnısse und Verhinderungen, 1978

25 J. Borley: Die Nachtschichtarbeit von Frauen, in: Bundesministerium a. a. O., S. 35 ff., S. 39.

26 G. Durig: Arr. 3 Abs. II, in: Th. Maunz. 11. a.: Grundgesetz, 1973, Rdn. 47.

27 Vgl. P. Drohsel: Die Lohndiskriminierung der Frauen, 1986.

28 M. Csillag/J. Eschinger: Frauennachtarbeirsverbot und Gleichbehandlung im EG-Raum, in: ZAS r992,

S. 17 ff., S. 20.

29 BVerfG, r BvL s1/86, s0/87 und, BvR 873/90, 761/91 vom 7.7.1992, EuGRZ 1992, S. 308 ff. 
machen würde ${ }^{3 \circ}$. Durch bloße Mithilfe « einer größeren Anzahl von Männern im Haushalt und ein paar "Ausnahmeregelungen « mehr zugunsten von mit Haus- und Erziehungsarbeit belasteten Menschen ist wenig gewonnen. Beides stellt - wic schon an der Begrifflichkeit ablesbar - die heutige gescllschaftliche Normalitätsstruktur nicht in Frage.

Für eine effektive Umsetzung des grundgesetzlichen Gleichberechtigungspostulats in sciner historisch intendierten Stoßrichtung ebenso wie in seiner funktional heute gebotenen Interpretation nicht nur als Diskriminierungs-, sondern auch als Gleichstellungsgebot zugunsten der Frauen"s sind folgende Konsequenzen zu ziehen:

- Individualrechtlich konzipierte Diskriminierungsverbotc können nur dort zugunsten der Frauen als Gruppe effektiv werden, wo Gleichheit zwischen den Geschlechtern im Sinne von Angleichung an die Position der Männer als Gruppe auch tatsächlich herstellbar ist. Sie allein können allerdings auch in diesem Fall nicht genug Breitenwirkung entfalten.

- Ausnahmeregelungen und geschlechtsspezifisches Schutzrecht haben dort ihre Existenzberechtigung, wo Gleich heit zwischen den Geschlechtern nicht herstellbar ist. Mit Sicherheit kann das jedoch nur im Bereich der biologischen Geschlechtsunterschiede, also im Zusammenhang mit Schwangerschaft und Geburt, angenommen wcrden. Weil dieses Recht tradierte Geschlechtsrollen zu konservicren ncigt, solte es daher auch nur auf diesen Bereich begrenzt angewendet werden.

- Zur Beseirigung struktureller Frauendiskriminierung bedarf es eines neuen gleichstellenden Rechts, das seine Zielgrößen nicht cinfach bei den Männern suchen und auf die Frauen übertragen darf. In einer umfassenden Betrachtung von Erwerbsbereich und sog. Privatbereich ist jeweils zu ermitteln, was eine gesellschaftich optimale neue Lösung für beide Geschlechter sein könnte, um cine Angleichung der Chancen dorthin zu befördern. Dafür müssen außerhalb des tradierten Antidiskriminierungsrechts neue Wege beschritten wcrden, dic auch eine Bezugnahme auf das Geschlecht gestatten, allerdings nur soweit und solange es für die Transformationsaufgabe unerläßlich ist.

\section{Das Nachtarbeitsverbot zwischen Diskriminierung, Schutz und Gleichstellung der Frau}

Für den EuGH war ausschlaggebendes Argument zur Ablehnung des Frauennachtarbeitsverbots, daß cs für Frauen im Wettbewerb um Arbeitsplätze mit Männern ein diskriminierender Nachteil sei, der sich nicht mehr durch alte Schutzgedanken rechtfertigen lasse. Doch die Wettbewerbsperspektive ist nicht so eindeutig, wie die EuGH-Entscheidung glauben macht. Keine Nachtarbeit leisten zu müssen, ist vom Blickpunkt der Lebensqualität her durchaus auch ein Privileg - solange Tagesarbeit als Alternative offensteht. Umgckehrt eröffnet die Abschaffung des Nachtarbeitsverbots Frauen nicht nur neue Arbeitsbereiche. Sie verschließt möglicherweise familiär besonders belasteten Frauen auch bisherige Arbeitsbereiche, weil diese Frauen nun kein Gegenrecht gegen ihren nächtlichen Einsatz mehr geltend machen könncn ${ }^{32}$. In

30 Siche hierzu die Perspektiven von Muckenberger u. a.: Niemand darf wegen seiner Arbeitszeitoptionen benachteiligt werden, in: Die Mitbesummung 6/1992, S. 8 ff.

31 Ausfuhrlich hierzu H. Pfarr: Quoten und Grundgesetz, 1988, S. $27 \mathrm{ff}$.; S. Raasch: Frauenquoten und Mannerrechte, 1991, S. $119 \mathrm{ff}$; V. Slupik: Die Entscheidung des Grundgesetzes fur Parıtit im Geschlechterverhälınis, 1988 .

32 Vgl. I. Rowhanı: Die Nacht den Frauen? in: Bundesministersum a. a. O., S.61 ff., S.69f. 
der Praxis zu beobachten sind beide Entwicklungstendenzen. In Japan verwandelte sich die Halbleiterindustrie mit Einführung der vollkontinuierlichen Produktion unter Fortgeltung des Frauennachtarbeitsverbots innerhalb von nur zehn Jahren bis 1982 von einer $80 \%$ igen Frauenindustrie zu einer $80 \%$ igen Männerindustrie ${ }^{33}$. Wäre es allerdings ohne die Frauen nicht gegangen, weil auf dem Arbeitsmarkt zu wenig Männer zur Verfügung gestanden hätten, darf nach den überall geltenden, wirtschaftspolitisch motivierten Durchbrechungen des Nachtarbeitsverbots ${ }^{34}$ allerdings vermutet werden, daß die japanischen Frauen auch hier aufgrund einer Ausnahmeregclung hätten bleibcn können. Umgekehrt zeigen dic Zugangsschwierigkeiten, die Frauen in den attraktiven Bereichen der gewerblich-technischen Berufe noch immer haben, selbst wenn dort keine Nachtarbeit zu leisten ist ${ }^{35}$, daß der Verweis auf das Nachtarbeitsverbot die Wettbewerbsnachteile von Frauen auf dem Arbeitsmarkt keinesfalls hinrcichend crklärt. Auch in Schweden und der DDR, in deren Rechtssystem es kein Frauennachtarbeitsverbot gab und gibt, war und ist der Arbeitsmarkt genau wie in der Bundesrepublik Deutschland geschlechtsspczifisch segmentiert ${ }^{36}$.

Schutzeffekt und Diskriminicrungsrichtung sind also im Fall des Frauennachtarbeitsverbots nicht eindeutig zu bestimmen. Sie wechseln je nach den arbeitsmarktpolitischen Vorzeichen. Den Frauen werden aufgrund ihrer Durchsetzungsschwäche auf dem Arbeitsmarkt als doppelt belastete Arbeitskräfte in Regelfall die Prämissen von außen gesetzt. Mit oder ohne Nachtarbeitsverbot können sie ihre Arbeitsmarktwettbewerbsfreiheit nicht ausnutzen, solange die strukturelle Diskriminierung auf der Basis heutiger geschlechtsspezifischer Arbeitsteilung fortdauert. Diesem Problem allerdings stellt sich der EuGH wie auch das bisherige EG-Recht ${ }^{37}$ gerade nicht.

Der VfGH begründet seine Entscheidung für die Beibehaltung des Frauennachtarbeitsverbots mit dem Schutzaspekt. Seiner Ansicht nach sind die Wettbewerbsnachteile, die Frauen durch das Nachtarbeitsverbot eventuell auf dem Arbeitsmarkt erleiden, im Interesse ihres Schutzes vor noch weitergehenden und gefährlicheren Nachteilen hinzunehmen ${ }^{38}$.

Ob dieser Schutz tatsächlich positiv zu bewerten ist, bleibt jedoch auch außerhalb seiner tradierte Gesellschaftsrollen konservierenden Langzeiteffekte und neben der Tatsache seiner rein wirtschaftlich orientierten Durchlöcherung fraglich. Die mit Hausarbeit belastete Frau befindet sich nämlich in einer Zwickmühle, dic auch der VfGH sieht: Einerseits lehnen gerade Frauen Nachtarbcit aus familiären Gründen besonders stark ab. Andererseits jedoch bietet Nachtarbeit für viele Frauen faktisch die einzige oder doch günstigste Möglichkeit, überhaupt erwerbstätig zu sein. Empirisch feststellbar ist einerseits eine besonders kritische Haltung verheirateter Frauen und insbesondere von Frauen mit Kindern zur Nachtarbeit. Aus familiären Gründen wird gerade diese Arbeitsform abgelehnt. Andererseits sind faktisch dann doch mehr Frauen mit kleinen Kindern als andere bereit, nachts zu arbeiten ${ }^{39}$. Dem-

33 1. Lenz: Frauenarbeits-Futurismus-Tango, in: Beitrage zur feministıschen Theorie und Praxis $9 / 101983$. S. 7 fff., S. 89 ,

34 So auch Th. Blank/H. Dicderich: Das Ende des Nachtarbeitsverbots? in: ArbuR 1992, S. I6s ff., S. : 66 .

35 Vgl. J. Strauß: Junge Frauen in gewerblich-technischen Berufen, 1986

36 Vgl. M. Srerner/G. Furse Mellstrom: Das schwedische Experiment, $198 \mathrm{~s}$, S. 19 ff.; W/nkler a. a. O., S. ss fif.

37 S. Raasch: Perspektiven fur die Gleichberechtigung im EG-Binnennarkt '92, in: KJ I990, S.62 ift, S. 64 .

38 VfGH, in: Bundesministcrium a. a. O., S. A 43 ; VfSlg. $11.774 / 1988,5.870$.

39 Borley, a. a. O., S. 48 f.; V. Peykan: Zur Situation von Nachtscirichtarbcicerimen, in: Bundesministeriun, a.a. O., S. 1 fff., S. so; Sesfert/Stolz-Willig, a.a.O., S. is9. 
entsprechend arbeiten in Schweden, wo es kein spezielles Frauennachtarbeitsverbor gibt, zwar auch mehr Männer als Frauen nachts, aber ein größerer Anteil der Frauen als der Männer tut dieses sogar regelmäßig ${ }^{+0}$. Neben Teilzeitarbeit scheint sich in Schwcden Nachtarbeit, oft ebenfalls in Teilzeit, als zweites Instrument für Fraucn herausgebildet zu haben, Beruf und Familie so zu verbinden, daß am Tag gemeinsame Zeit mit den Kindern übrigbleibt.

Die Nachfrage der Frauen nach Nachtarbeit ist nicht nur Ergcbnis eines übcrmäßigen wirtschaftlichen Drucks, wie der VfGH annimmt, und auch nicht nur fehlenden Kinderbetrcuungsmöglichkeiten geschuldet ${ }^{4 t}$. Sie entspricht auch einem Bedürfnis der Frauen, zu arbeiten und dennoch mit ihren Kindern Tageszeit zusammen zu verbringen. Denn geradc in Sclıweden ist die Kinderbetreuungssituation extrem günstig. Nicht ein Verbor der Nachtarbeit für Frauen, sondern eine Vcrändcrung der Normalarbeitszeit tagsüber dürfte dementsprechend diejenige Antwort sein, die diese Widersprüche in Wünschen und Verhalten der Frauen angemcssen aufnimmt.

Nach Ansicht des VfGH ist es allcin Aufgabe des Gesetzgebers, abzuwägen zwischen noch erforderlichem Schutz, der dann jedoch möglicherwcise dic überkommene Rollenverteilung zwischen den Geschicchtern verfestigc, und Angleichung dcr Lebensverhältnisse von Frauen und Männern auf Kosten eines verläßlichen Schutzes der gegenwärtig Betroffencn ${ }^{42}$. Dcm liegt eine Verfassungsbestimmung zugrunde, die mit Art. 7 Abs. x B-VG in Österreich lediglich einen allgemeinen Gleichheitsgrundsatz cnthält, der vom VfGH im Sinne eines Differenzierungsverbots ähnlích wie Art. 3 Abs. I und Art. 3 Abs. 3 GG interpretiert wird ${ }^{43}$. Allerdings hat der VfGH anderenorts bereits vertreten, nur solche Ungleichbchandlungen zwischen den $\mathrm{Ge}-$ schlechtern seien sachlich gerechtfertigt, die wenigstens in Richtung eines Abbaus der Unterschiedc wirken würden. Ungleichheiten, denen diese Funktion nicht zukomme und die die bestehonden Unterschiede zwischen den Geschlechtern noch vertieften, seien verfassungswidrig ${ }^{44}$. An dieses Postulat erinnerte sich der VfGH im Zusammenhang mit dem Nachtarbeitsverbot 1988 bzw. 1992 nicht, so daß sich seine Abwägungen nur zwischen Schutz und Gleichbchandlung bewegten, den Gleichstellungsaspekt in der Geschlechterfrage jedoch nicht berücksichtigten. VfGH und EuGH gleichen sich darin, ihre rechtlichen Entscheidungsgrundlagen gleichstellungspolitisch begrenzt zu definieren. Unter Ausklammern des gleichstellungspolitischen Aspektes bewcrten sie dann allerdings mit Schutz vor Doppelbclastung bzw. Wettbewerbsgleichheit unterschiedliche Aspekte höher.

Auch das EG-Rccht enthält jedoch eine Frauen glcichstellendc Dimension über die rein wettbewerbsorientierten normativen Grundlagen des Lohngleichheitssatzes aus Art. I 9 EWGV und die EG-Gleichbehandlungsrichtlinien hinaus. Zwar geht es zu weit, direkt aus der Zielsetzung des Art. 1 9 EWGV und den EG-Gleichbehandlungsrichtlinien eine EG-rechtliche Verpflichtung aller Staatsgewalt zur umfassenden Verwirklichung der Gleichstellung der Geschlechter abzuleiten's. Mit seiner Defrenne III-Entscheidung $x 978$ hat der EuGH zumindest aus umfassenderen men-

40 Vgl. G. Pettersson (Hg.): Zeit-Puzzlc, 1990, S. 34.

41 So aber Borley, a. a. O., S. 51 .

42 VFGH, Bundesministerium, a. a. O., S. A 44

43 Vgl. Csillag/Eichinger, a. a. O., S. 21 Fn. 39 m.w. N.

44 VfGH, EuGRZ. 1991, S. 484 ff., S. 48 s und 486 jeweils m. w. N.

45 Ausführlich dagegen S. Raasch: Perspekuven, a. 3. O., S.64 ff.; ciniur N. Colneric: Vorlagepflichr nacls EG-Recht bei Normkontrolle fur Frautnquote, in: BB 1991 , S. 118 ; Pfarr, a. a. O., S. 66 ff.; vorsichtiger M. Inde: Vorlagepflicht des Bundesverfassungsgerichts an den EuGH bei der Normkontrolle uber Friuenquote, in: BB 1992, S. 489 . 
schenrechtlichen Überlegungen heraus einen allgemeinen Grundsatz des Gemeinschaftsrechts angenommen, die auf dem Geschlecht beruhenden Diskriminierungen auch über die in Art. 19 EWGV allein geregelre Lohngleichheit hinaus zu beseitigen ${ }^{46}$, ihm jedoch keine unmitcclbare Rechtsgeltung gegenüber nationalem Recht verliehen. Obwohl die seiner Argumentation zugrunde liegenden völkerrechtlichen Verträge auch einc erweiterte gleichstellungspolitische Dimension dieses Menschenrechts ${ }^{47}$ und damit auch des gemeinschaftsrechtlichen Grundsatzes nahelegen und obwohl sozialpolitische Programme der EG ebenfalls in diese Richtung weisen, beharrt der EuGH bisher auf seiner rein wetrbewerbsmäßig ausgerichteten Gleichberechtigungsinterpretation.

Nach Ansicht des BVerfG darf das Nachtarbeitsverbot für Frauen als wetrbewerbswidrige Geschlechtsdiskriminierung weder fortbestehen wie nach Ansicht des VfGH noch einfach fallen wie nach Ansicht des EuGH. Es wurde für mit der Verfassung für unvereinbar erklärt und der Gesetzgeber aufgefordert, eine Neuregelung zu schaffen, die beide Geschlechter angemessen vor den negativen Folgen von Nachtarbeit schüt$\mathrm{ze}^{\mathrm{t}^{8}}$. Die rechtlichen Konsequenzen bis zu dieser niche terminlich fixierten Neuregelung werden damit komplizierter ${ }^{49}$. Die Entscheidung läuft vorübergehend auf ein Recht der Frauen auf Nachtarbeit kombiniert mit einer persönlichen Verweigerungsmöglichkeit hinaus, welches sich unter Gleichbehandlungsgesichtspunkren auch auf Männer erstrecken müßre, soweit diese dicsclbc Schutzbedürftigkeit wie Fraucn aufweisen. Das BVerfG argumentiert, daß geschlechtsspezifische Differenzierungen nur zulässig seien, wenn sie an Probleme anknüpften, die ihrer Natur nach nur bei einem Geschlccht auftreten könnten, oder aber die Gleichberechtigung der Geschlechter für die Zukunft durchsetzen wollten.

In seiner Nachtarbeitsentscheidung faßt das BVerfG noch einmal in erfreulicher Klarheit zusammen, was seiner Ansicht nach Inhalt des Gleichberechtigungsgebots aus Art. 3 Abs. 2 GG ist. Dabei trifft es zum ersten Mal eine deutliche Aussage dazu, daß Arr. 3 Abs. 2 und Art. 3 Abs. 3 1. Alt. GG unterschiedliche Regclungsgehalte haben, und bestimmt auf dieser Basis deren Verhältnis zueinander. Besonders in dicsen strukturierenden Passagen der Entscheidung liegt, weit über die Beurteilung des Nachtarbeitsverbots hinaus, das Zukunftsweisende der Entscheidung ${ }^{\circ}$. Demnach ist Arr. 3 Abs. 3 GG lediglich ein Diskriminierungsverbot, während Art. 3 Abs. 2 GG cinen darübcrhinausreichenden Regelungsgehalt hat. Der Gleichberechtigungssatz wolle für die Zukunft die Gleichberechtigung der Geschlechter durchsetzen, zielc auf einc Angleichung der Lebensverhältnisse und verbiete die Verfestigung überkommener Rollenverteilungen zwischen den Geschlechtern durch staat-

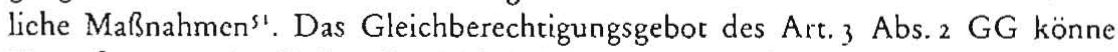
Verstöße gegen das Verbot der Diskriminierung wegen des Geschlechts rechrfertigen, ohne dieses allerdings im Fall des Nachtarbeitsverbors auch tatsächlich zu tun ${ }^{52}$. Denn das Nachtarbeitsverbot sei wegen seiner geschlechtsrollenkonservierenden Wirkung gerade kein Anwendungsfall des Art. 3 Abs. 2 GG.

Damit hat sich das BVerfG grundsätzlich geöffnet für eine differenziertcre Gleich-

46 EuGH Slg. 1978 , S. 1365 ff., S. 1379.

47 Siehe hicrzu Raasch: Frauenquoten, a. a. O., S. $172 \mathrm{ff}$

48 BVertG EuGRZ 1992, S. $22 \mathrm{f}$.

49 Siehe hierzu N. Colneric: Konsequenzen der Nachtarbcitsverbotsurtcile des EuGH und des BVerfG, in: N7.A 1992, S. 393 ff.; J. Zmarslik: Auswirkungen des Urteils des Bundesverfassungsgerichts über die Unverein barkeit des Naehtarbeitsverbots fur Arbeiterinnen mit Art. 3 GG, in: DB 1992, S. 680ff.

so Ebenso Blanke; Diederich, a. a. O., S. 170 ff.

S1 BVerfG, EuGRZ 1992, S. 21.

ऽ2 BVeriG, EuGRZ 1992, S. 22. 
stcllungspolitik über verschiedene Wege, wie sie unter 2. entwickelt wurde und allein auch gegen strukturelle Diskriminierung Wirkung verspricht. Für die Frage, ob zur Verwirklichung der Gleichstellung der Frau im Erwerbsleben Frauenquoten zulässig sind's, bedeuten diese Klarstellungen einen wichtigen Schritt in Richtung auf gcrichtliche Anerkennung ihrer Verfassungskonformität. Zwar bleibt weiterhin offen, ob die Verwirklichung der Gleichstellung der Frau ein Verfassungsauftrag oder nur eine Verfassungsaufgabe ist und wie weit Frauen dafür subjektive Rechte gegeben werden. In jedem Fall stützt aber das BVerfG dic in der Literatur entwickelte Position $^{54}$, daß das zugunsten von Frauen und Männern wirkende Diskriminierungsverbot des Art. 3 Abs. 3 GG im Konflikrfall zugunsten der gleichstellenden Zielsetzung des Art. 3 Abs. 2 GG zurücktritt. Eines Rückgriffs auf das Sozialstaatspostulat, wie ihn noch Benda zugunsten aktiver Gleichstellungspolitik für erforderlich hielts', bedarf es nicht. Die Position des OVG NW, der in Art. 3 Abs. 2 GG enthaltene Gleichberechtigungssatz schließe eine Differenzierung wegen des Geschlechts über Quoten vom Ansatz her ebenso aus wie das Diskriminierungsverbot in Art. 3 Abs. 3 $G^{s 6}$, findet in der heutigen Rechtsprechung des BVerfG zum Nachtarbeitsverbot keine Stütze mehr.

53 Vgl. Vorlagebeschluß des OVG NW 12. Senat 23. 10. 1990, DVBI 1991, S. 118ff., aufgehoben durch Beschluß vom 10. 4. 1992, DVBI. 1992, S. 1372; OVG NW 6. Scnat, 7.7.1992 A 7.6 B 71 3 und 809/92. $\$ 4$ Vgl. R. Francke u. a.: Frauenquoten in der offenthchen Ausbildung, 199r, S. 95 ff.: Pfarr, a. 2. O., S. 78 ff.; Raasch, Frauenquoten, a. a. O., S. 242 ff.

ss Benda a. a. O., S. 137 ff. und 145 ff.

s6 OVG NW NJW 1989 , S. 2560 . 